\title{
Development of measurement system for powder flowability based on vibrating capillary method
}

\section{$\operatorname{AUTHOR}(S)$ :}

Jiang, Yanbin; Matsusaka, Shuji; Masuda, Hiroaki; Qian, Yu

\section{CITATION:}

Jiang, Yanbin ... [et al]. Development of measurement system for powder flowability based on vibrating capillary method. Powder Technology 2009, 188(3): 242-247

\section{ISSUE DATE:}

2009-01-10

URL:

http://hdl.handle.net/2433/85002

\section{RIGHT:}

Copyright (c) 2008 Elsevier B.V.; This is not the published version. Please cite only the published version.; この論文は出版社版でありません。引 用の際には出版社版をご確認ご利用ください。 


\title{
Development of measurement system for powder flowability based on vibrating capillary method
}

\author{
Yanbin Jiang $^{1}$, Shuji Matsusaka ${ }^{2 *}$, Hiroaki Masuda ${ }^{2}$ and Yu Qian ${ }^{1}$
}

1. School of Chemical Engineering, South China University of Technology, Guangzhou 510640, China

2. Department of Chemical Engineering, Kyoto University, Kyoto 615-8510, Japan

\begin{abstract}
An automatic measurement system for evaluating powder flowability based on the vibrating capillary method has been studied experimentally. The vibration amplitude was controlled by a computer, and the vibration acceleration and the mass of particles discharged from the capillary were obtained by digital processing. The measurement time was 2 min and the amount of the powder required for each measurement was less than a few grams. To characterize the dynamic powder flowability, evaluation factors were developed. A series of experiments was conducted using powders with mass median diameters from 5.2 to $58.2 \mu \mathrm{m}$, showing that this measurement system can fully evaluate the powder flowability.
\end{abstract}

Keywords: Powder flowability; Vibration; Capillary; Automatic measurement

* Corresponding author.

E-mail address: matsu@cheme.kyoto-u.ac.jp. (S. Matsusaka) 


\section{Introduction}

The study of powder flow is one of the most important things in powder handling processes. Evaluation of powder flowability is often required for quality control of powder products as well as stable process operation [1]. The powder flowability is not an inherent material property, but is the result of the combination of many factors, e.g. particle size, shape, particle interaction, packing fraction, flow rate, temperature, humidity, and electrostatic charge. Thus, it is not easy to theoretically estimate the powder flowability.

The powder flowability has been characterized by a number of conventional methods, such as measuring the angle of repose, Carr's flowability index, Jenike flow factor, and the value determined using the Hall flowmeter [1-4]; however, technical knowledge and skills are necessary to perform successfully. Also, there are other problems, including poor repeatability and indirect evaluation of dynamic behavior. To solve these problems, other methods were proposed, e.g. using a twisted blade [5], a vibratory feeder [6], and an avalanche testing instrument [7-9]. However, proper experimental methods are still needed to detect slight differences in flowability of fine powders. In general, a small sensing area is desirable for raising sensitivity, and well-defined evaluation factors are required for accurate evaluation. Recently, a method for evaluating the powder flowability has been proposed by Jiang et al. [10, 11]. This method is based on the analysis of powder flow in a vibrating capillary, which was studied for the first time by Matsusaka et al. [12, 13], further by Yang and Li [14], and Yang and Evans [15, 16].

In the present work, we develop an automatic measurement system for evaluating the powder flowability based on the vibrating capillary method, and study the performance of this system through experiments using sample powders with different mass median diameters.

\section{Experimental}

\subsection{Experimental apparatus}

Fig. 1 shows a schematic diagram of the experimental apparatus. The basic components of this system were almost the same as those shown in previous paper of the authors [10]. A glass tube, $100 \mathrm{~mm}$ long and $6 \mathrm{~mm}$ inner diameter, with a capillary tube, $50 \mathrm{~mm}$ long and $1.2 \mathrm{~mm}$ inner diameter, was held vertically, and a hopper was fixed on top of the glass tube, which was kept full with powder during measurement. The capillary was vibrated in the horizontal direction by a 
piezoelectric vibrator. The mode of the vibration was controlled by a computer, and the input electric AC voltage for the vibration can be sinusoidal, rectangular, triangular, saw-tooth, or other shapes. In this experiment, a sinusoidal waveform was used because of the advantage of a natural vibration. The frequency and amplitude of vibration can be automatically increased or decreased. The vibration at the end of the capillary was measured by a laser vibrometer (LV-1300, Ono-sokki Co. Ltd.) which was a non-contact measurement system. The mass of particles discharged from the capillary was measured by a digital balance with a resolution of 0.1 mg. All the data were recorded to the computer through an A/D converter (NR-350, Keyence Co.) at the intervals of $2 \mathrm{~s}$.

\subsection{Experimental conditions}

Since the vibration amplitude of the capillary depends on the vibration frequency, we measured the resonance points beforehand. The first resonance frequency of this system was $330 \mathrm{~Hz}$, and the resonance wave was very stable. Therefore, the vibration frequency was fixed at the resonance point for all the experiments. The vibration amplitude can be changed by the applied voltage. To characterize the dynamic powder flowability in a short period, we increased the vibration amplitude from 0 to $130 \mu \mathrm{m}$ at a constant rate for 2 min and obtained the mass of particles discharged from the vibrating capillary tube as a function of time elapsed. From the results, the relationship between powder flow rate and vibration acceleration can be analyzed. This method is simple and convenient, but can have some errors because of the hysteresis of powder flow. To investigate the hysteresis, the vibration amplitude should be increased and decreased. Furthermore, to evaluate the stability of powder flow, an experiment was conducted at a constant vibration acceleration. Sample powder was put into the hopper, and vibrated at an amplitude of $130 \mu \mathrm{m}$ to fill the capillary before measuring the powder flowability. The operation was repeated three times to evaluate the repeatability. All the experiments were conducted under room conditions (temperature: $17-20{ }^{\circ} \mathrm{C}$, relative humidity: 35-45\%). The electric charge of particles discharged from the vibrating capillary was less than $1.5 \times 10^{-5} \mathrm{C} / \mathrm{kg}$, which was the same as the value in the previous paper [10]. Therefore, van der Waals force exerted on the particles is thought to be dominant in this experiment. 


\subsection{Sample powders}

Two kinds of fused silica particles (Denki Kagaku Kogyo K.K.) and nine kinds of polymethylmethacrylate (PMMA) particles (MBX series, Sekisui Plastics Co., Ltd.) were used in this work. The properties of sample powders listed in Table 1 are the particle density $\rho_{\mathrm{p}}$, the mass median diameter $D_{\mathrm{p} 50}$ and the geometric standard deviation of particle diameter distribution $\sigma_{\mathrm{g}}$

Table 1. Properties of sample powders

\begin{tabular}{cccc}
\hline Material & $\rho_{\mathrm{p}}\left(\mathrm{kg} / \mathrm{m}^{3}\right)$ & $D_{\mathrm{p} 50}(\mu \mathrm{m})$ & $\sigma_{\mathrm{g}}(-)$ \\
\hline \multirow{2}{*}{ Fused silica } & 2200 & 13.6 & 1.6 \\
& & 30.4 & 1.6 \\
& & 5.2 & 1.4 \\
& & 6.7 & 1.5 \\
& & 12.3 & 1.6 \\
Polymethyl- & \multirow{2}{*}{1200} & 13.8 & 1.6 \\
methacrylate & & 17.3 & 1.4 \\
(PMMA) & & 27.6 & 1.4 \\
& & 38.9 & 1.5 \\
& & 47.9 & 1.5 \\
& & 58.2 & 1.5 \\
\hline
\end{tabular}

2.4. Observation of powder flow and packing structure of particles in the vibrating capillary

A high-speed microscope camera (Fastcam-Max, Photron Ltd.) was used to observe the powder flow in the vibrating capillary. The frame rate was $4000 \mathrm{fps}$ and the images were recorded to the computer. Also, a micro-focus X-ray CT system (SMX-100CT, Shimadzu Co.) was used to observe the packing structures of the particles in the capillary. The images of the packing structure were taken under non-vibrating condition after experiment using the vibrating capillary.

\section{Results and discussion}

\subsection{Observation of powder flow in the vibrating capillary}

The mechanism of the powder flow in the vibrating capillary has been discussed elsewhere $[10,13]$. The gravitational force is the driving force to discharge particles from the capillary, while adhesive forces and friction forces between particles and the wall are hindrances. When 
the capillary is horizontally vibrated, a small clearance is formed between particles and the wall. As a result, the interaction forces are reduced, then particles move down through the capillary [10, 16]. The formation of the clearance is prominent where the void fraction is high and particles have larger inertia. Fig. 2 shows snapshots of the powder flow at the end of the vibrating capillary. The capillary was initially almost full with the particles. When vibration was applied, a space was observed at the end of the capillary, and the position of the space was changed with the vibration. As particles flow out of the capillary, the average void fraction increases and the particles are loosely connected with each other, therefore, the particles can easily move in the horizontal and vertical directions. This phenomenon was seen in all the measurements.

In general, vibration may increase the particle packing density, and consequently makes the discharge of the particles difficult [10]. Therefore, the packing structure of the particles in the capillary should be observed beforehand. Fig. 3 (a) shows a profile of powder flow rate as a function of the vibration acceleration $\alpha\left(=a(2 \pi f)^{2}\right)$, where $a$ is the vibration amplitude and $f$ is the vibration frequency. When the vibration acceleration exceeds a certain value, the powder begins to flow (Fig. 3 (a) (ii)). The flow rate first increases with the vibration acceleration and then decreases (Fig. 3 (a) (ii) $\rightarrow$ (iv)); i.e. there is an upper limit. Fig. 3 (b) shows the CT images at the point $20 \mathrm{~mm}$ from the end of the capillary. They were obtained by the following procedure; when the vibration acceleration reached a selected value, the vibration was stopped and, to avoid the change of the packing structure, the capillary was carefully detached and set into the measuring cell of the micro-focus X-ray CT system. After taking a CT image, the capillary was again set to the vibration unit and the vibration acceleration was increased from the above selected value to the next selected value. In the same way, 4 images were obtained. In Fig. 3(b), voids in the particle layer look black. For smaller vibration acceleration, the black area is inhomogeneously distributed in the packing structure (see Fig. 3 (b) (i) and (ii)); however, the black area decreases with increasing vibration acceleration, and the particles are homogeneously and densely packed (see Fig. 3 (b) (ii) $\rightarrow$ (iv)). Therefore, the variation of the mass flow rate in the profile of the flowability includes the effect of the packing structure.

\subsection{Repeatability}

Repeatability is one of the key specifications for powder flowability measurements. Fig. 4 
shows three measurements and average value of the flowability profile of each sample powder (PMMA). The profiles have different features depending on the mass median diameter of the sample powder; however, it is found that the repeatability is reasonably good.

\section{3. Effect of mass median diameter on powder flowability}

Fig. 5 shows all the experimental results obtained using the PMMA sample powders with different mass median diameters. Each curve indicates the average of three measurements of the mass of particles discharged from the vibrating capillary as a function of time elapsed. This figure indicates that the mass of particles increases with the mass median diameter; i.e. the flowability increases with the diameter. Also, it was found that the amount of particles required for each measurement was less than a few grams. Fig. 6 shows the mass flow rate obtained by digital processing of the data shown in Fig. 5. Here, the mass flow rate, which is the average value of the time interval (2 s), is expressed as a function of the vibration acceleration. The mass flow rate is zero under no vibration, but begins to increase after exceeding a certain vibration acceleration. This vibration acceleration is defined as the critical vibration acceleration. There is, however, an upper limit of the mass flow rate as mentioned in Section 3.1. Although these features can be seen for various powders, characteristic values depend on the mass median diameter.

\subsection{Critical vibration acceleration making powder flow}

The critical vibration acceleration is here determined as the value where the powder flow rate exceeds $2 \mathrm{mg} / \mathrm{s}$. Fig. 7 shows the relationship between the critical vibration acceleration and the mass median diameter. The error in the repeatability of the critical vibration acceleration was within $10 \%$. Since the critical vibration acceleration is related to the static properties such as static friction and adhesiveness, the decrease in the critical acceleration implies the increase in the flowability. The flowability defined by the critical acceleration increases with the mass median diameter. In particular, for fine particles less than $20 \mu \mathrm{m}$ in mass median diameter, the effect of the particle diameter on the flowability is prominent, thus, slight difference of the powder flowability can be detected. 


\subsection{Maximum mass flow rate}

The mass flow rate of particles is also related to the dynamic property, namely dynamic friction in packing structure. To characterize the dynamic flowability of each powder, we chose the maximum mass flow rate as a parameter to evaluate the complicated dynamic flowability. Fig. 8 shows the relationship between the maximum mass flow rate and the mass median diameter of the sample powders. It is found that the flowability defined by the maximum mass flow rate is well correlated with the mass median diameter.

\subsection{Flow hysteresis of powder flow}

Flow hysteresis is one of the important factors to evaluate the powder flow. To obtain the data of the hysteresis, experiments were carried out with increase and decrease in the vibration acceleration. The period of time is 2 min for each operation. As a typical example, shown in Fig. 9 are three measurements and the average of mass of particles discharged from the vibrating capillary. It is found that the repeatability is reasonably good, and a hysteresis can be seen at the vibration acceleration less than $200 \mathrm{~m} / \mathrm{s}^{2}$. When the vibration acceleration increases from zero, powder does not flow below the critical vibration acceleration, and powder flow rate increases sharply above the critical value. On the other hand, when the vibration acceleration decreases to zero, the powder keeps flowing even though the acceleration is zero. The hysteresis is probably caused by the experience of the packing condition in the capillary and also the difference between the static and dynamic friction.

\subsection{Stability of powder flow rate}

Stability is also an important factor to evaluate the powder flow. Fig. 10 shows the mass flow rate as a function of time elapsed. These measurements were carried out at a constant vibration acceleration $(f=75.4 \mu \mathrm{m})$ using powders of $D_{\mathrm{p} 50}=12.3,27.6$, and $58.2 \mu \mathrm{m}$. For smaller particles, the mass flow rate tends to fluctuate frequently (Fig. 10 (a)); however, the stability is better with increasing particle diameter (Fig. 10 (b) $\rightarrow$ (c)). The difference in the stability is probably due to the adhesiveness of particles or the ratio of the adhesive force to the gravitational force. 


\subsection{Comparison with the angle of repose}

It is interesting to compare these obtained data with those from a conventional method. Measurement of the angle of repose is widely used to characterize the powder flowability because of the simplicity and convenience. The angle of repose is related to the critical condition making powder flow. The present vibrating capillary method can evaluate the powder flowability from various view points, i.e. critical vibration acceleration making powder flow, mass flow rate as a function of vibration acceleration, hysteresis and stability of the powder flow. Among of them, we select the critical vibration acceleration because of the similarity to the definition of the powder flowability.

Fig. 11 shows the experimental results obtained with the two methods. For reference, the critical vibration amplitude as well as the critical vibration acceleration is given in the ordinate of the figure. From these results, it is found that there are similar features, i.e. both the critical vibration acceleration and the angle of repose decrease with the increase in mass median diameter. However, the variation of the angle of repose is very small $\left(<3^{0}\right)$ over $20 \mu \mathrm{m}$ in mass median diameter, thus, it will be difficult to evaluate slight difference. On the other hand, the variation of the critical vibration amplitude is sufficiently larger than the limit of the reliability $(1 \mu \mathrm{m})$. Therefore, the vibrating capillary method can have a high resolution in the flowability analysis.

\section{Conclusion}

We have developed an automatic measurement system for evaluating powder flowability based on the vibrating capillary method. The vibration was controlled by the computer and the motion of the capillary was measured by the non-contact measurement method. The mass of particles discharged from the capillary was measured by the digital balance. Following evaluation factors were obtained by digital processing: critical vibration acceleration making powder flow, mass flow rate as a function of vibration acceleration, hysteresis and stability of the powder flow. It was confirmed that the system can evaluate the dynamic flow of powders in a range from 5.2 to $58.2 \mu \mathrm{m}$ in mass median diameter, and have a high resolution in comparison with the conventional method based on the angle of repose. 


\section{Acknowledgement}

The authors gratefully acknowledge the support from Kyoto Prefecture Collaboration of Regional Entities for the Advancement of Technological Excellence, JST and National Scientific Fund of China (20536020, 20676046). The authors are also grateful to Denki Kagaku Kogyo K.K. and Sekisui Plastics Co., Ltd. for providing the sample powders and to Shimadzu Co. for obtaining the CT images.

\section{References}

[1] J. Schwedes, Review on testers for measuring flow properties of bulk solids, Granular Matter, 5 (2003) 1-43.

[2] H. Masuda, H. Yoshida, K. Iinoya, Statistical analysis of the Carr's flowability index, Journal of the Research Association of Powder Technology, Japan, 12 (1975) 209-216.

[3] K. H. Staffa, J. Jahn, N. Claussen, Flowability of powders under influence of vibrations, Powder Metallurgy International, 9 (1977) 20-23.

[4] S. Kamath, V. M. Puri, H. B. Manbeck, R. Hogg, Flow properties of powders using four testers -measurement, comparison and assessment, Powder Technology, 76 (1993) 277-289.

[5] R. Freeman, An insight into the flowability and characterization of powders, American Laboratory, Aug. 33 (2001) 13-16.

[6] S. N. Bhatachar, D. B. Hedden, A. M. Olsofsky, X. Qu, W. Hsieh, K. G. Canter, Evaluation of the vibratory feeder method for assessment of powder flow properties, International Journal of Pharmaceutics, 269 (2004) 385-392.

[7] B. H. Kaye, Fractal dimensions in data space; new descriptors for fine particle systems, Particle \& Particle Systems Characterization, 10 (1993) 191-200.

[8] F. Lavoie, L. Cartilier, R. Thibert, New methods characterizing avalanche behavior to determine powder flow, Pharmaceutical Research, 19 (2002) 887-893.

[9] B. C. Hancock, K. E. Vukovinsky, B. Brolley, I. Grimsey, D. Hedden, A. Olsofsky, R. A. Doherty, Development of a robust procedure for assessing powder flow using a commercial avalanche testing instrument, Journal of Pharmaceutical and Biomedical Analysis, 35 (2004) 979-990.

[10] Y. Jiang, S. Matsusaka, H. Masuda, T. Yokoyama, Evaluation of flowability of composite 
particles and powder mixtures by a vibrating capillary method, Journal of Chemical Engineering of Japan, 39 (2006) 14-21.

[11] S. Matsusaka, H. Masuda, Y. Jiang, M. Yasuda, Powdery-granular material fluidity evaluating device and method, PCT/JP2006/308223, WO 2006/115145.

[12] S. Matsusaka, M. Urakawa, H. Masuda, Micro-feeding of fine powders using a capillary tube with ultrasonic vibration, Advanced Powder Technology, 6 (1995) 283-293.

[13] S. Matsusaka, K. Yamamoto, H. Masuda, Micro-feeding of a Fine Powder Using a Vibrating Capillary Tube, Advanced Powder Technology, 7 (1996) 141-151.

[14] Y. Yang, X. C. Li, Experimental and analytical study of ultrasonic micro powder feeding, Journal of Physics D: Applied Physics, 36 (2003), 1349-1354.

[15] S. F. Yang, J. R. G. Evans, Computer control of powder flow for solid freeforming by acoustic modulation, Powder Technology, 133 (2003), 251-254.

[16] S. F. Yang, J. R. G. Evans, Acoustic initiation of powder flow in capillaries, Chemical Engineering Science, 60 (2005), 413-421.

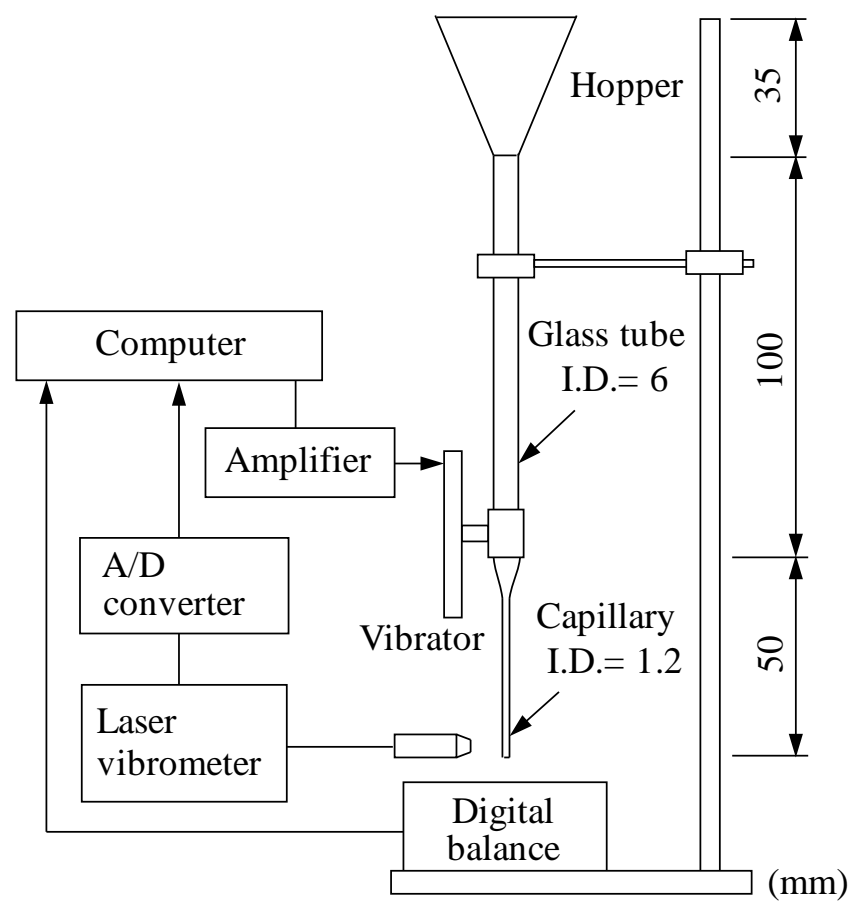

Fig. 1. A schematic diagram of the experimental apparatus 


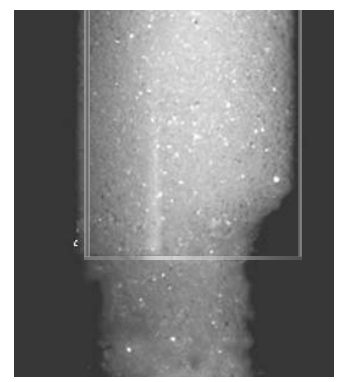

(a) $0 \mathrm{~ms}$

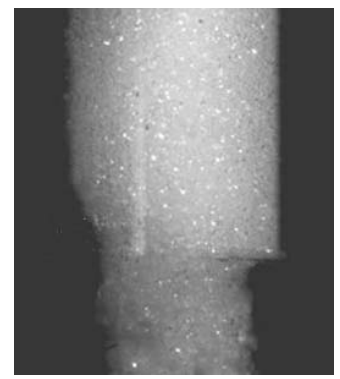

(d) $1.5 \mathrm{~ms}$

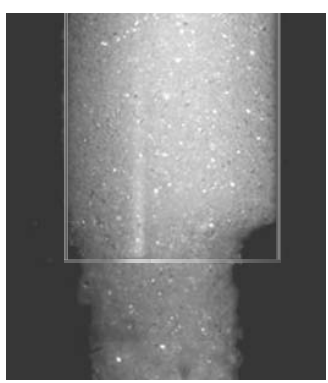

(b) $0.5 \mathrm{~ms}$

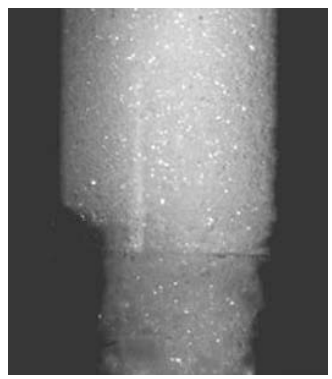

(e) $2 \mathrm{~ms}$

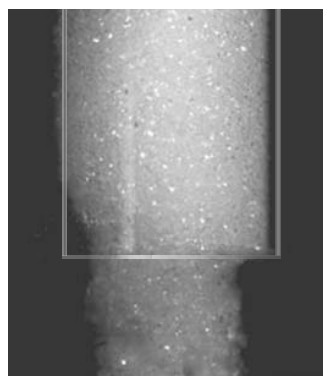

(c) $1 \mathrm{~ms}$

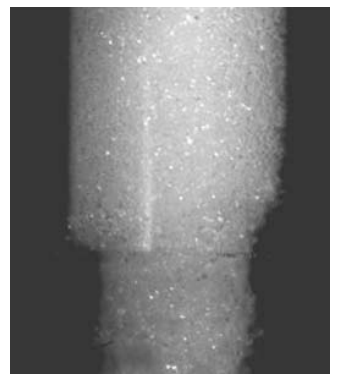

(f) $2.5 \mathrm{~ms}$

$\stackrel{\longmapsto}{0.5 \mathrm{~mm}}$

Fig. 2. Snapshots of the powder flow at the end of the vibrating capillary made of glass (Silica particles: $\mathrm{MMD}=30.4 \mu \mathrm{m}$, vibration frequency: $f=330 \mathrm{~Hz}$, vibration amplitude: $a=130 \mu \mathrm{m}$ )

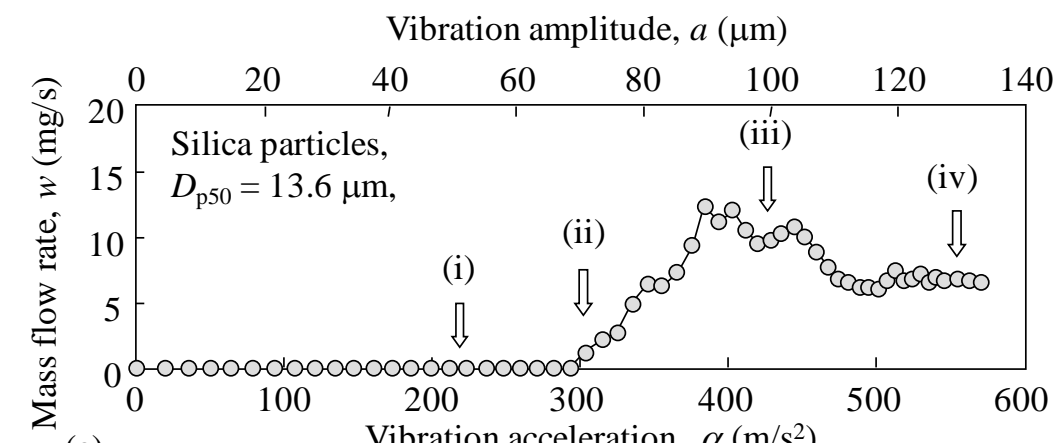

(a)

Vibration acceleration, $\alpha\left(\mathrm{m} / \mathrm{s}^{2}\right)$

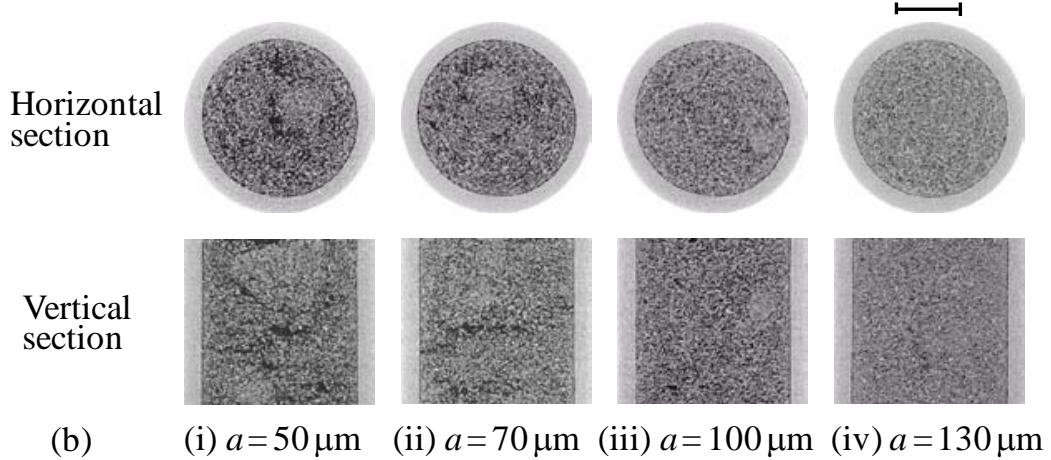

Fig. 3. Packing structures of particles in the vibrating capillary tube $(\mathrm{f}=330 \mathrm{~Hz})$

(1) Profile of mass flow rate; (2) Images of packing structure, which were taken with a micro-focus X-ray CT system 


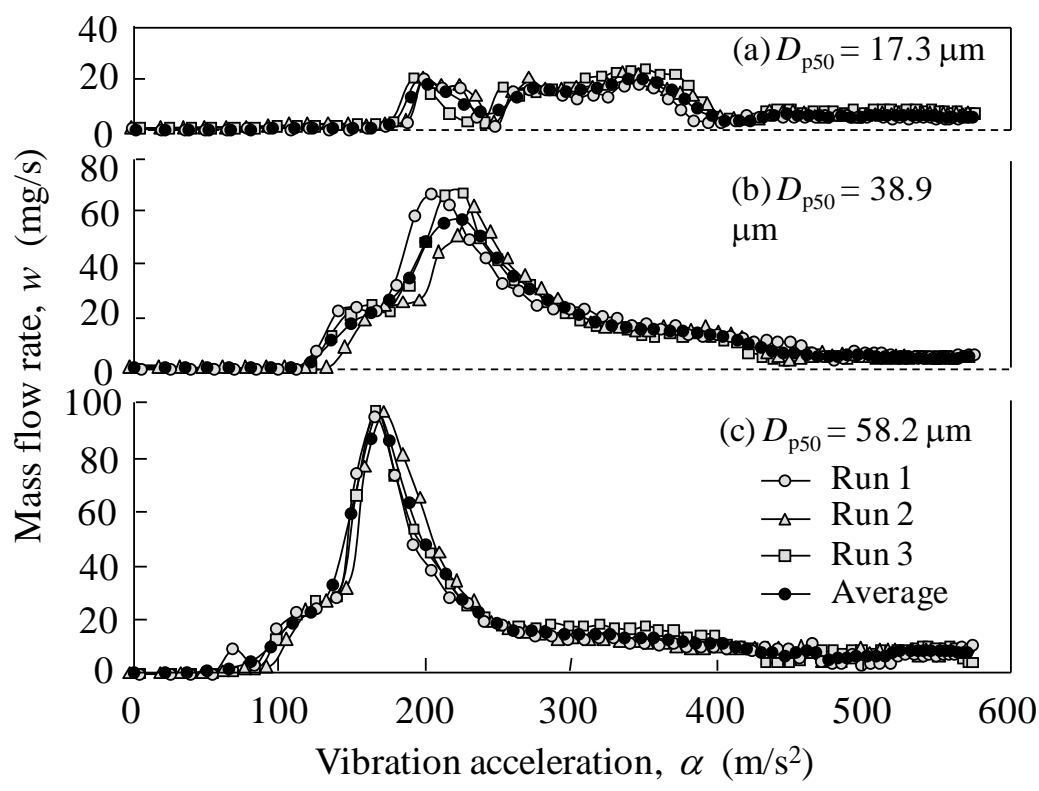

Fig. 4 Repeatability of flowability profile (PMMA particles)

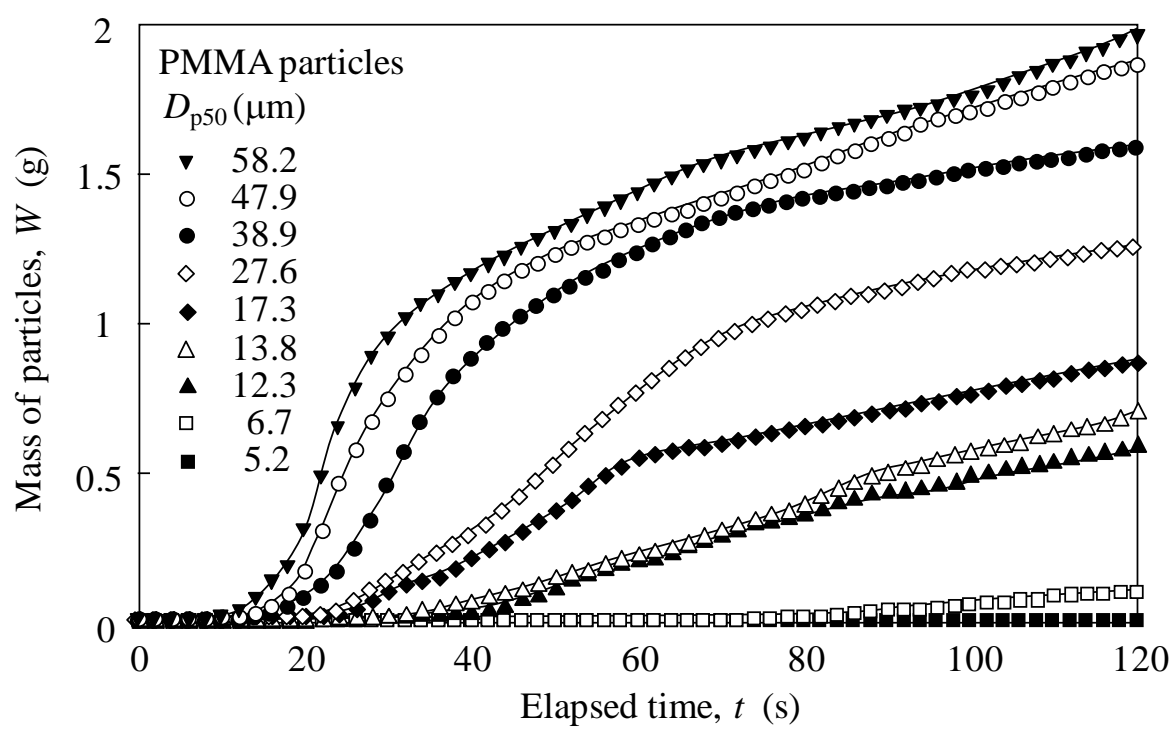

Fig. 5 Mass of particles discharged from the vibrating capillary 


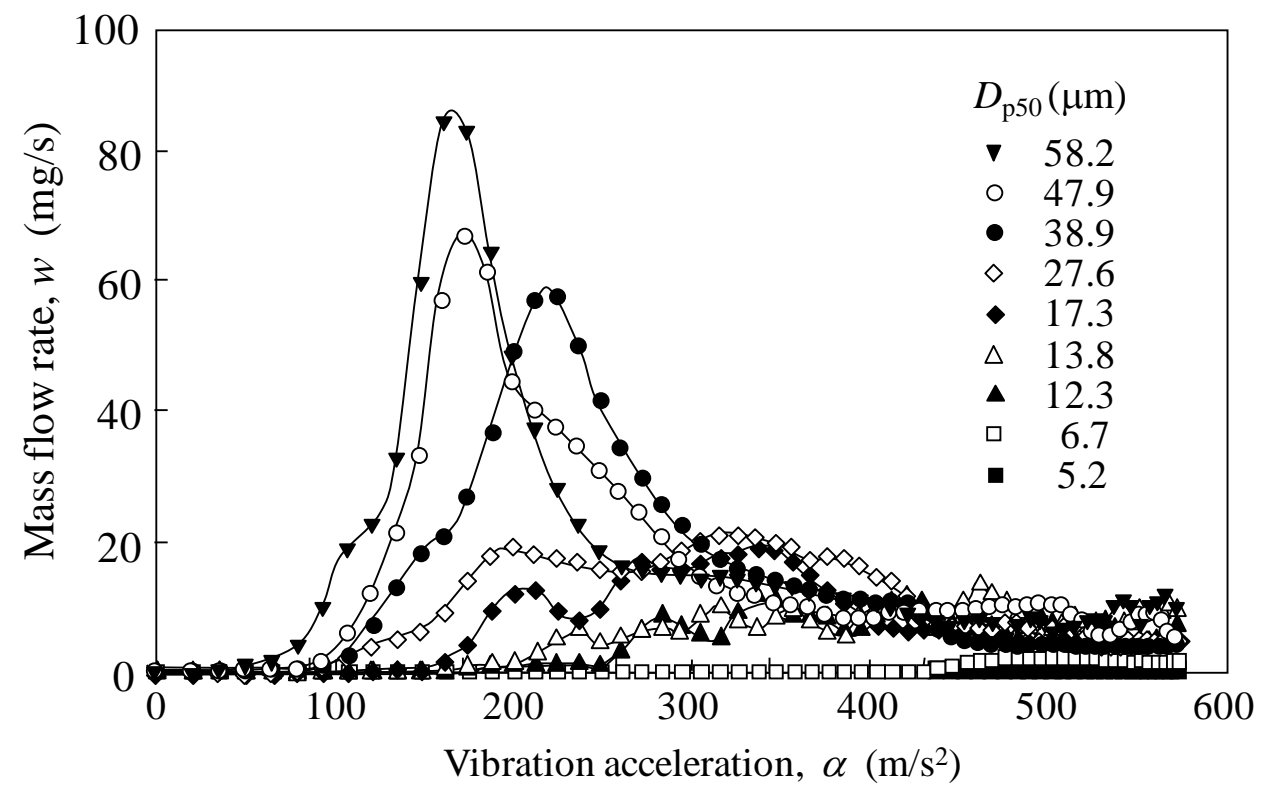

Fig. 6 Profiles of powder flowability

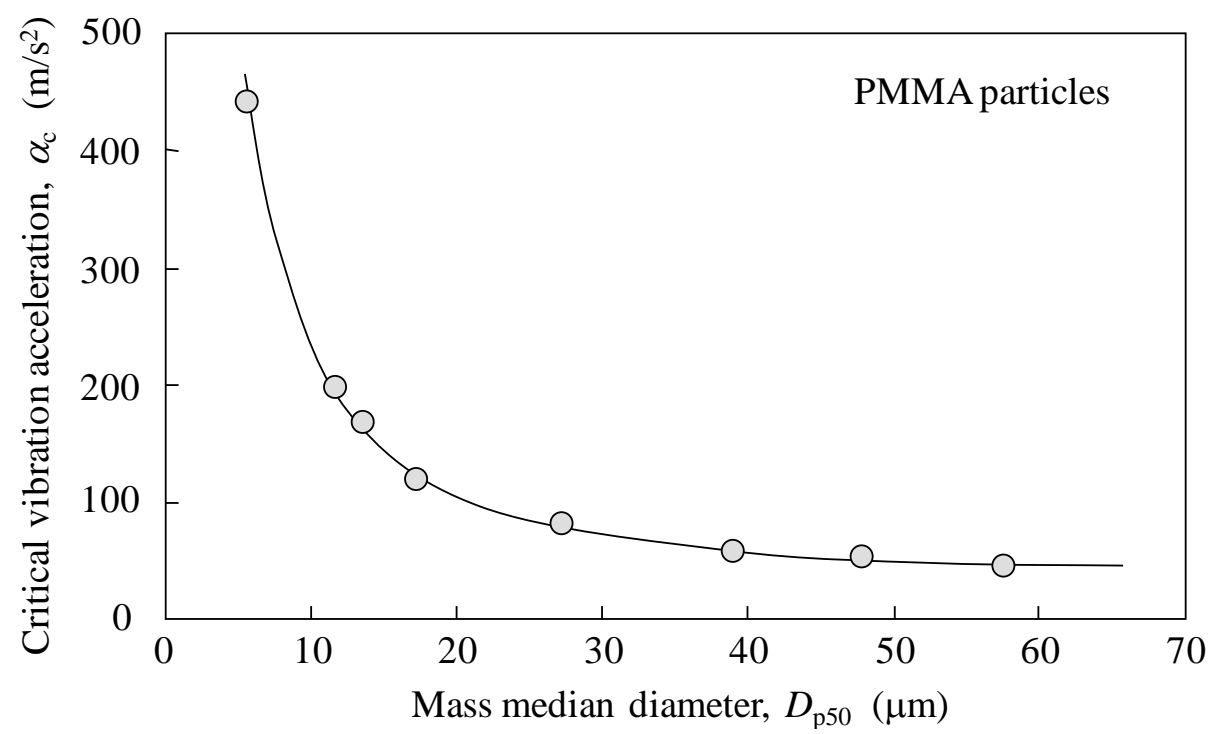

Fig. 7 Relationship between critical vibration acceleration and mass median diameter 


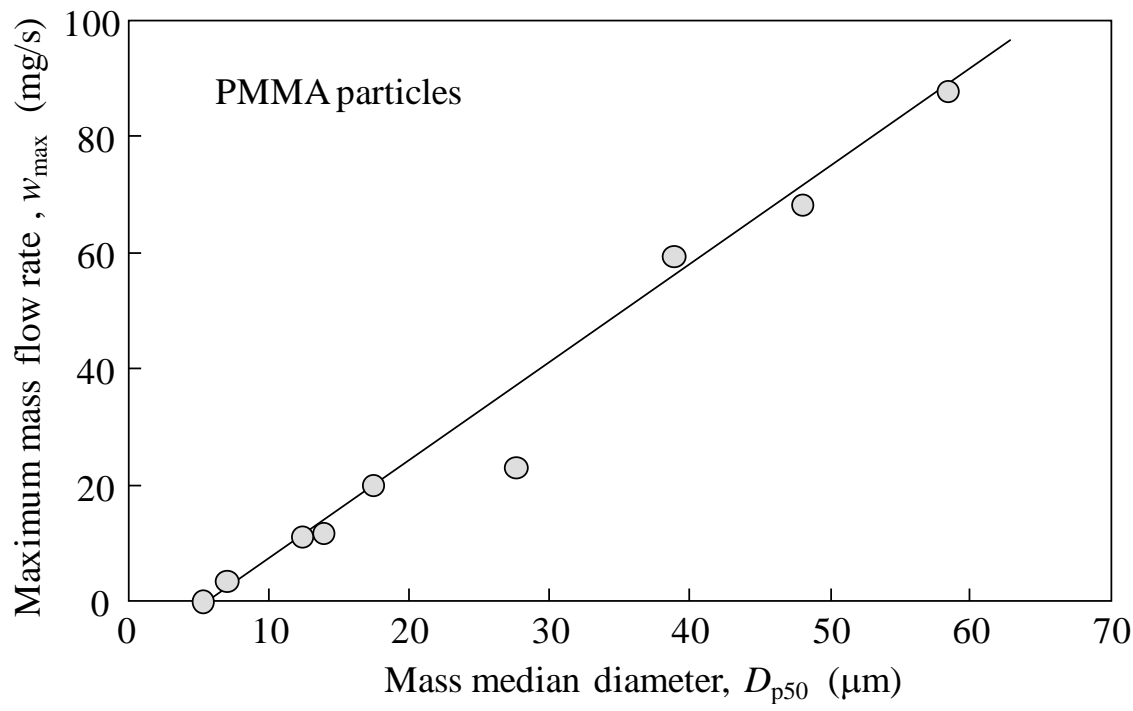

Fig. 8 Relationship between maximum mass flow rate and mass median diameter

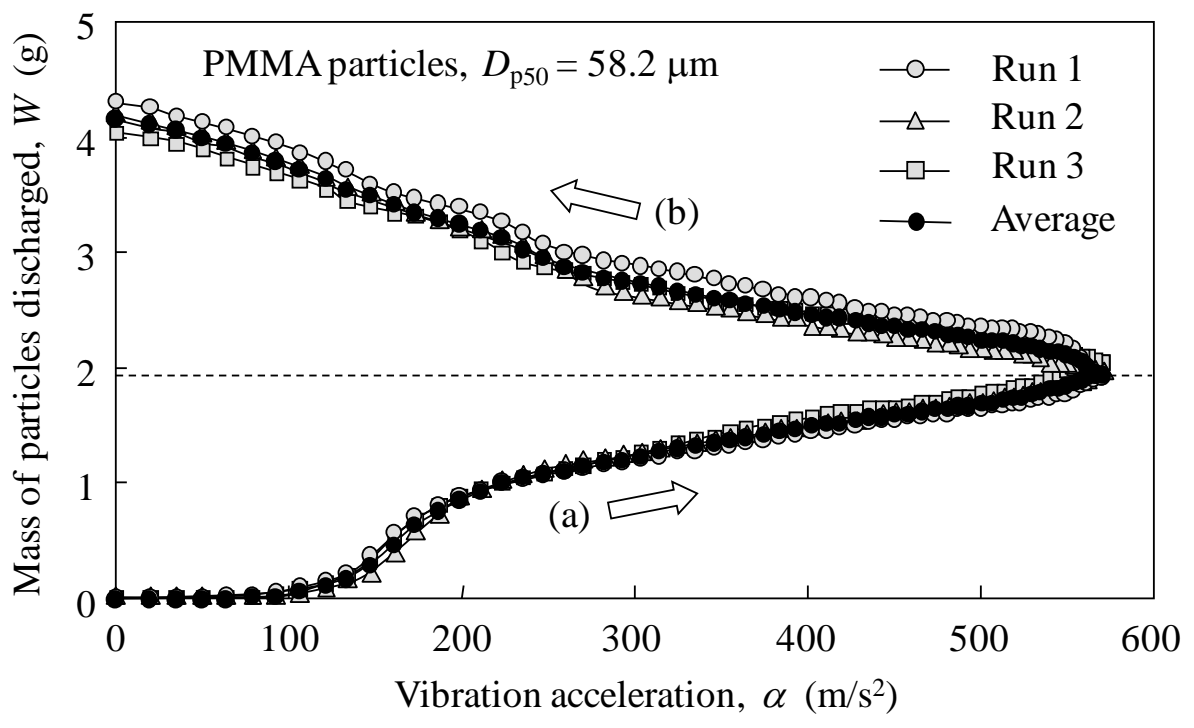

Fig. 9 Hysteresis of particle flow ; (a) increase and (b) decrease in vibration amplitude 


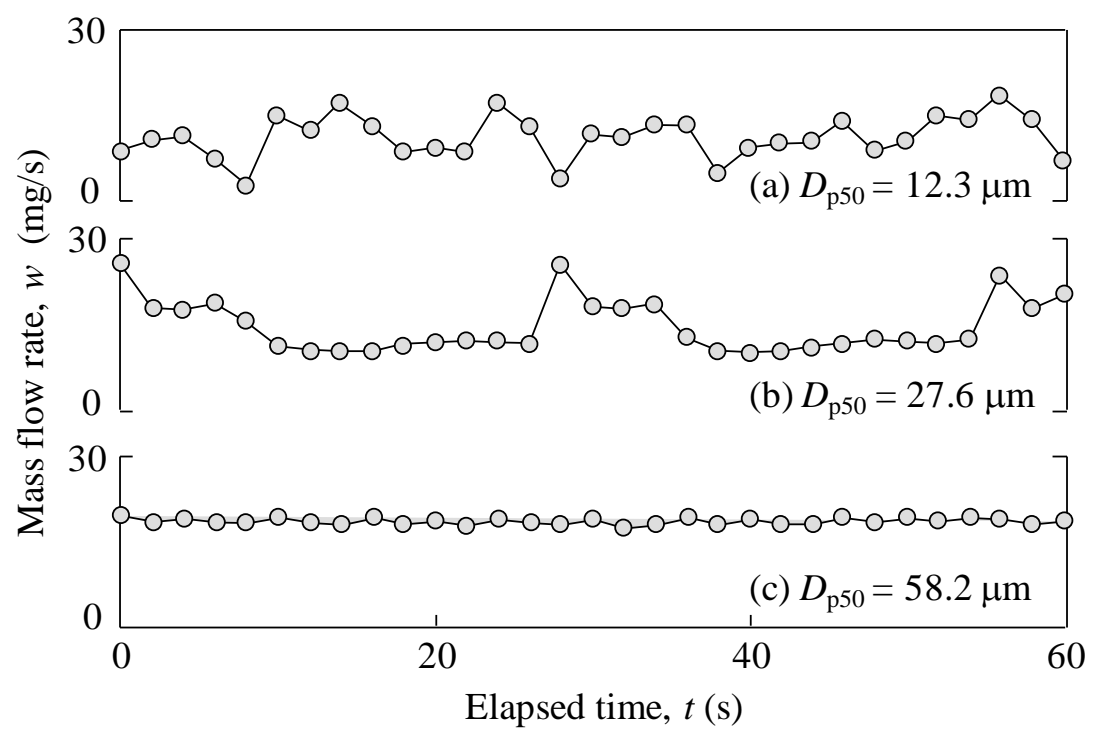

Fig. 10 Stability of flowability (PMMA particles, vibration acceleration: $\alpha=324 \mathrm{~m} / \mathrm{s}^{2}$ )

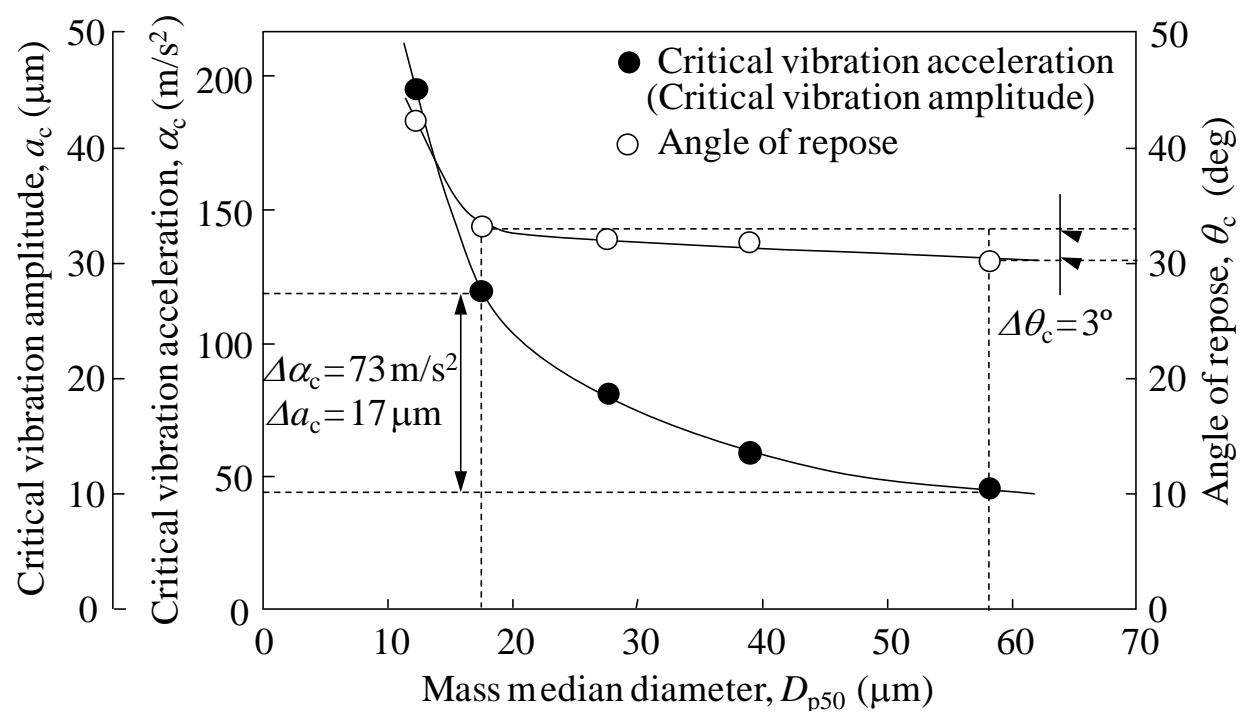

Fig. 11 Comparison between critical vibration acceleration and angle of repose (PMMA particles) 